\title{
HIGHER ORDER COMPACT FINITE DIFFERENCE METHOD FOR THE SOLUTION OF 2-D TIME FRACTIONAL DIFFUSION EQUATION
}

\author{
Muhammad Usman ${ }^{*}$, Noor Badshah¹, Fazal Ghaffar ${ }^{2}$ \\ ${ }^{1}$ Department, of Basic Science \& Islamiyat UET, Peshawar, Pakistan. \\ ${ }^{2}$ Department, of Mathematics, Government Jehanzeb Post Graduate College Saidu Sharif Swat, KPK, Pakistan. \\ *Corresponding Author Email: usmanss2010@gmail.com
}

This is an open access article distributed under the Creative Commons Attribution License, which permits unrestricted use, distribution, and reproduction in any medium, provided the original work is properly cited.

\section{ARTICLE DETAILS}

\section{Article History:}

Received 04 November 2017 Accepted 02 December 2017

Available online 18 January 2018

\begin{abstract}
The main purpose of this study is to work on the solution of two-dimensional time fractional diffusion equation In this research work we apply the HOC scheme to approximate the second order space derivative. To obtain a discrete implicit scheme, Grunwald-Letnikov descritization is used in sense to approximate the RiemannLiouville time fractional derivative. The scheme thus obtained is based on block pentadiagonal matrix and each matrix has five-point stencil in order to reduce the computational cost we use AOS method. In AOS method, before taking the average of two solutions first we split the n-dimensional problems into a sum of n-one dimensional problem.
\end{abstract}

\section{KEYWORDS}

FDE's, HOC iterative scheme, AOS scheme, Convergence, Fourier analysis

\section{INTRODUCTION}

FDEs are the generalizations of ordinary order differential equations. In recent years fractional differential equations (FDEs) are mostly studied and get more attraction for their development and research. FDEs have a lot of importance in countless fields of science and technology such as in biological, physical, geological and financial systems. Recently it has become a strong tool which is used for a good modeling control of processes and signal processing for various fields of science and engineering. Fractional diffusion equation has the main physical purpose, to describe phenomenon of irregular diffusion processes. It is interesting to develop some schemes that efficiently solve fractional differential equations.

As we know that many schemes have been proposed to solve FDEs theoretically such as Laplace, Fourier transform method, Green function method, but most problems cannot be solved analytically and hence in this regard it is very remarkable to solve fractional differential equations numerically $[1,2]$. The whole conversation about the fractional differential equations are presented in [2]. Numerous numerical method have been planned for the solution of time or spatial FDEs, for instance Finite difference method for time fractional diffusion equation which are presented in some studies, generated explicit and semi-implicit schemes for the solution of fractional order PDEs [3,4]. Many researchers wished for a model and encountered that the fractional derivatives are more accurate [5-7]. They also inquired a finite difference scheme and analyze the stability condition for fractional reaction- subdivision equations [8]. The stability criterion for an implicit scheme were discussed in [9]. Recently a numerical scheme is proposed for fractional sub divisional equation and to analyze accuracy by local Fourier analysis scheme using multigrid method [10-14]. They developed explicit and implicit FDM for fractional reaction sub divisional equation $[15,16]$. Also, an implicit numerical technique was introduced for modified irregular Subdivision equation [17]. The compact difference scheme explained for second order space derivatives [17-19]. Recently compact finite difference method developed for fractional diffusion equation [20-23]. The paper is presented in sense to show the solution of 2-D time fractional diffusion equation using higher order compact finite difference method (CFDM).

To the best of our knowledge there are no such reports with higher order scheme in terms of space variable. Hence, so it is interesting to investigate the higher order CFDM for FDEs. Compact methods are the higher order methods in which the coefficient matrix of the linear system of equations is in the form of pantadiagonal or block diagonal that can be solved easily by high order compact(HOC) scheme, here we want to talk about 2-D time fractional diffusion equation as the model problem with a source term as given in $[8,24]$.

$$
\begin{aligned}
\frac{\partial^{\gamma} \mathrm{v}(\mathrm{t}, \mathrm{x}, \mathrm{y})}{\partial \mathrm{t}^{\gamma}}= & { }_{0} D_{t}^{1-\gamma}\left[K_{1} \frac{\partial^{2} \mathrm{v}}{\partial x^{2}}+\mathrm{K}_{2} \frac{\partial^{2} \mathrm{v}}{\partial y^{2}}\right]+f(t, x, y) . \\
& (x, y) \epsilon\left(L, L_{0}\right) \times\left(L_{1}, L_{2}\right) . \quad 0<t<T
\end{aligned}
$$

Where $k_{1}$ and $k_{2}$ are positive constant and ${ }_{0} D_{t}^{1-\gamma} u(1>\gamma>$ $0)$ Represents the Rieman-Liouville fractional derivative of the function $f(t, x, y$,$) defined as, i.e, \quad{ }_{0} D_{t}^{1-\gamma} u=\frac{1}{\Gamma(\gamma)} \cdot \frac{\partial}{\partial t} \int_{0}^{t} \frac{v(\tau, x, y)}{(t-\tau)^{1-\gamma}} d \tau$.

\subsection{Mathematical formulation of the Model problem.}

We present the numerical solution of equation (1) as follows, using Taylor's series to get the second order central difference scheme which is as, $\frac{1}{h^{2}} \delta_{x}^{2} v=\frac{1}{h^{2}}\left(v_{i+1, j}^{k}-2 v_{i, j}^{k}+v_{i-1, j}^{k}\right)$ which represents the second order approximation to $u_{x x}$. while on the other side the higher order operator in compact scheme is obtained from the approximation of as in [25],

$$
\begin{aligned}
\left(\frac{\partial v}{\partial x}\right)_{i, j}=\left(\frac{2 \mu_{x}}{h} \cdot \frac{\sinh ^{-1} \frac{\delta_{x}}{2}}{\left(1+\frac{\delta_{x}^{2}}{4}\right)^{\frac{1}{2}}}\right) v_{i, j} & \\
& =\frac{\mu}{h}\left(\delta_{x}-\frac{1^{2}}{3 !} \delta_{x}^{3}+1^{2} \cdot \frac{3^{2}}{5 !} \delta_{x}^{5}-\cdots\right) v_{i, j} .
\end{aligned}
$$

the average operator $\mu_{x}$ is described as $\mu_{x} v_{i, j}=\frac{1}{2}\left(v_{1+\frac{1}{2}, j}+v_{1-\frac{1}{2}, j}\right)$

$\left(\frac{\partial^{2} v}{\partial x^{2}}\right)_{i, j}=\left(\frac{2}{h} \cdot \sinh ^{-1} \frac{\delta_{x}}{2}\right)^{2} v_{i, j}=\frac{\mu}{h}\left(\delta_{x}^{2}-\frac{1}{12} \delta_{x}^{4}+\cdot \frac{1}{90} \delta_{x}^{6}-\frac{1}{560} \delta_{x}^{8}+\right.$

-..) $v_{i, j}$

(4).

To get higher order operator we multiply the operator in equation (3) by 
equation (4) as in [25]

$$
\begin{aligned}
\left(\frac{\partial^{4} v}{\partial x^{4}}\right)_{i, j}=\frac{\partial^{2}}{\partial x^{2}} \cdot \frac{\partial^{2} v_{i, j}}{\partial x^{2}} & \\
& =\frac{1}{h^{4}}\left(\delta_{x}^{4}-\frac{1}{6} \delta_{x}^{6}+\frac{21}{720} \delta_{x}^{8}\right. \\
& +\cdots) v_{i, j}
\end{aligned}
$$

Now after rearranging the terms we have,

$$
\begin{gathered}
\left(\frac{\partial^{2} v_{i, j}^{k}}{\partial x^{2}}\right)=\left(\frac{\delta_{x}^{2} v_{i, j}^{k}}{h^{2}\left(1+\frac{\delta_{x}^{2}}{12}\right)}\right)+\frac{h^{4}}{240} \frac{\partial^{4} v_{i, j}^{k}}{\delta x^{4}} \\
+O\left(h_{x}^{6}\right)
\end{gathered}
$$

we consider as follows,

$\left(\frac{\partial^{4} v_{i, j}^{k}}{h^{4}\left(1+\frac{\delta_{x}^{2}}{12}\right)}\right)_{i, j}=\frac{1}{h^{4}}\left(\delta_{x}^{4}-\frac{1}{12} \delta_{x}^{6}+\frac{1}{144} \delta_{x}^{8}+\cdots\right) v^{k}{ }_{i, j}$

Taking equation (5) and neglecting higher order terms we have.

$\left(\frac{\partial^{4} v_{i, j}^{k}}{h^{4}\left(1+\frac{\delta_{x}^{2}}{12}\right)}\right)=\left(\frac{\partial^{4} v_{i, j}^{k}}{\partial x^{4}}\right)$

To get the higher order approximation for $\frac{\partial^{2} v_{i, j}^{k}}{\partial x^{2}}$ to maintain accuracy of sixth order and pentadiagonal of the scheme we play with equation (8) in equation (6), i-e.,

$\left(\frac{\partial^{2} v_{i, j}^{k}}{\partial x^{2}}\right)=\left(\frac{\delta_{x}^{2} v_{i, j}^{k}}{h^{2}\left(1+\frac{\delta_{x}^{2}}{12}\right)}\right)+\frac{h^{4}}{240} \frac{\partial_{x}^{4} v_{i, j}^{k}}{\left(1+\frac{\delta_{x}^{4}}{12}\right)}+O\left(h_{x}^{6}\right)$ similarly, for $y$ direction the same procedure can be chase,

We descritized the Riemann-Liouville fractional derivative by using the idea of Grunwald-Litnikove estimate as in [2] is as below,

${ }_{0} D_{t}^{1-\gamma} f(t)=\frac{1}{\tau^{1-\gamma}} \sum_{\ell=0}^{\left[\frac{t}{\tau}\right]} \xi_{\ell}^{(1-\gamma)} f(t-\ell \tau)+o\left(\tau^{p}\right)$, where $\xi_{\ell}^{(1-\gamma)}$ represents the coefficient of the function and is defined as, $\sum_{l=0}^{\infty} \xi_{\ell}^{(1-\gamma)} \omega^{l}$

i-e., $\omega_{0}^{\alpha}=1, \omega_{l}^{\alpha}=\left(1-\frac{\alpha+1}{\ell}\right) \omega_{l-1}^{\alpha}, l \geq 1$

For simplification these coefficient will be denoted by, $\quad \lambda_{\ell} \equiv \omega_{\ell}^{(1-\gamma)}=$ $(-1)^{\ell}\left(\begin{array}{c}1-\gamma \\ \ell\end{array}\right), \ell=0,1, \ldots n$

The HOC scheme for equation (1) can be shown as for $k=$

$1,2,3 \ldots N, i=0,1,2 \ldots N x+1, j=0,1,2 \ldots N y+1$ as in [20] where

$N_{x}+1$ and $N_{y}+1$ are positive numbers. $\frac{v_{i, j}^{k}-v_{i, j}^{k-1}}{\tau}=$

$K_{\gamma} \frac{1}{h^{2} \tau^{1-\gamma}} \sum_{l=0}^{k} \lambda_{l}\left[\frac{\delta_{x}^{2}}{1+\frac{1}{12} \delta_{x}^{2}}+\frac{\delta_{y}^{2}}{1+\frac{1}{12} \delta_{y}^{2}}+\frac{h_{x}^{2} \delta_{x}^{4}}{240\left(1+\frac{1}{12} \delta_{x}^{2}\right)}+\frac{h_{y}^{2} \delta_{y}^{4}}{240\left(1+\frac{1}{12} \delta_{y}^{2}\right)}\right] v_{i, j}^{k-l}+f_{i, j}^{k}$

$$
\begin{aligned}
v_{i, j}^{0}=w\left(x_{i}, y_{j}\right), v_{0, j}^{k}= & \emptyset_{1}\left(y_{j} \cdot t_{k}\right), \quad v_{N_{x}+1, j}^{k}=\emptyset_{2}\left(y_{j}, t_{k}\right), \quad v_{i, 0}^{k} \\
& =\varphi_{1}\left(x_{i}, t_{k}\right), \quad v_{i, N_{y}+1}=\varphi_{2}\left(x_{i}, t_{k}\right) k=1,2,3 \ldots N, \quad i \\
& =0,1,2 \ldots N x+1 \quad j=0,1,2, \ldots N y+1
\end{aligned}
$$

$V_{i, j}^{k}$ represents the approximate solution of $v_{i, j}^{k}$ which shows that $v_{i, j}^{k}=$

$V_{i, j}^{k}+O\left(h^{6}\right)$,now multiplying both sides of equation (9) by (1+ $\left.\frac{1}{12} \delta_{x}^{2}\right)\left(1+\frac{1}{12} \delta_{y}^{2}\right)$ and applying the scaling parameter $\mu_{0}=\frac{K_{\gamma} \tau^{\gamma}}{h^{2}}$ then rearranging and taking $\lambda_{0}=1$, we have

$\left\{\begin{array}{c}\left(1+\left(\frac{1}{12}-\mu_{0}-\frac{\mu_{0} h^{2} \delta_{x}^{2}}{240}\right) \delta_{x}^{2}+\left(\frac{1}{12}-\mu_{0}-\frac{\mu_{0} h^{2} \delta_{y}^{2}}{240}\right) \delta_{y}^{2}\right. \\ \left.+\left(\frac{1}{144}-\frac{\mu_{0}}{6}-\frac{\mu_{0} h^{2} \delta_{x}^{2}}{240}-\frac{\mu_{0} h^{2} \delta_{y}^{2}}{240}\right) \delta_{x}^{2} \delta_{y}^{2}\right) V_{i, j}^{k}\end{array}\right)-\left(1+\frac{1}{12} \delta_{x}^{2}+\frac{1}{12} \delta_{y}^{2}+\frac{1}{144} \delta_{x}^{2} \delta_{y}^{2}\right) V_{i, j}^{k-1}$

$=\mu_{0} \sum_{l=0}^{k} \lambda_{l}\left(\begin{array}{c}\delta_{x}^{2}+\delta_{y}^{2}+\frac{\delta_{x}^{2} \delta_{y}^{2}}{6}+\left(1+\frac{1}{12} \delta_{y}^{2}\right) \frac{h^{2} \delta_{x}^{4}}{240} \\ +\left(1+\frac{1}{12} \delta_{x}^{2}\right) \frac{h^{2} \delta_{y}^{4}}{240}\end{array}\right) v_{i, j}^{(k-l)}+\tau\left(1+\frac{1}{12} \delta_{x}^{2}\right)\left(1+\frac{1}{12} \delta_{y}^{2}\right) f_{i, j}^{k}$

$v_{i, j}^{0}=w\left(x_{i}, y_{j}\right), v_{0, j}^{k}=\emptyset_{1}\left(y_{j} \cdot t_{k}\right), \quad v_{N_{x}+1, j}^{k}=\emptyset_{2}\left(y_{j}, t_{k}\right)$, $v_{i, 0}^{k}=\varphi_{1}\left(x_{i}, t_{k}\right), \quad v_{i, N_{y}+1}=\varphi_{2}\left(x_{i}, t_{k}\right)$

$k=1,2,3 \ldots N, \quad i=0,1,2 \ldots N x+1$

$j=0,1,2, \ldots N y+1$

Now after applying the operators and rearranging the terms we will get the following discritized form,

$$
\begin{aligned}
& e_{0} v_{i+2, j-1}^{k}+e_{0} v_{i+2, j+1}^{k}+e_{0} v_{i-2, j+1}^{k}+e_{0} v_{i-2, j-1}^{k} \\
& +e_{0} v_{i+1, j+2}^{k}+e_{0} v_{i-1, j+2}^{k}+e_{0} v_{i-1, j-2}^{k}+e_{0} v_{i+1, j-2}^{k} \\
& +d_{0} v_{i+2, j}^{k}+d_{0} v_{i-2, j}^{k}+d_{0} v_{i, j+2}^{k}+ \\
& d_{0} v_{i, j-2}^{k}+c_{0} v_{i+1, j+1}^{k}+c_{0} v_{i+1, j-1}^{k}+c_{0} v_{i-1, j+1}^{k} \\
& +c_{0} v_{i-1, j-1}^{k}+b_{0} v_{i+1, j}^{k}+b_{0} v_{i-1, j}^{k}+b_{0} v_{i, j+1}^{k} \\
& +b_{0} v_{i, j-1}^{k}+a_{0} v_{i, j}^{k}=e_{1} v_{i+2, j-1}^{k-1}+e_{1} v_{i+2, j+1}^{k-1} \\
& +e_{1} v_{i-2, j+1}^{k-1}+e_{1} v_{i-2, j-1}^{k-1}+e_{1} v_{i+1, j+2}^{k-1}+e_{1} v_{i-1, j+2}^{k-1} \\
& +e_{1} v_{i-1, j-2}^{k-1}+e_{1} v_{i+1, j-2}^{k-1}+d_{1} v_{i+2, j}^{k-1}+d v_{i-2, j}^{k-1} \\
& +d_{1} v_{i, j+2}^{k-1}+d_{1} v_{i, j-2}^{k-1}+c_{1} v_{i+1, j+1}^{k-1}+c_{1} v_{i+1, j-1}^{k-1} \\
& +c_{1} v_{i-1, j+1}^{k-1}+c_{1} v_{i-1, j-1}^{k-1}+b_{1} v_{i+1, j}^{k-1}+b_{1} v_{i-1, j}^{k-1}+b_{1} v_{i, j+1}^{k-1}+b_{1} v_{i, j-1}^{k-1} \\
& +a_{1} v_{i, j}^{k-1} \\
& +\boldsymbol{\tau}\left[\begin{array}{c}
a_{2} f_{i, j}^{k}+b_{2} f_{i+1, j}^{k}+b_{2} f_{i-1, j}^{k}+b_{2} f_{i, j+1}^{k}+b_{2} f_{i, j-1}^{k} \\
+c_{2} f_{i+1, j+1}^{k}+c_{2} f_{i+1, j-1}^{k}+c_{2} f_{i-1, j+1}^{k}+c_{2} f_{i-1, j-1}^{k}
\end{array}\right]+12 \sum_{l=2}^{k} \lambda_{l}\left[a_{3} v_{i, j}^{k-l}\right. \\
& +b_{3} v_{i+1, j}^{k-l}+b_{3} v_{i-1, j}^{k-l}+b_{3} v_{i, j+1}^{k-l} \\
& +b_{3} v_{i, j-1}^{k-l}+c_{3} v_{i+1, j+1}^{k-l}+c_{3} v_{i+1, j-1}^{k-l}+c_{3} v_{i-1, j+1}^{k-l} \\
& +c_{3} v_{i-1, j-1}^{k-l}+d_{3} v_{i+1, j+2}^{k-l}+d_{3} v_{i+1, j-2}^{k-l}+d_{3} v_{i-1, j+2}^{k-l} \\
& +d_{3} v_{i-1, j-2}^{k-l}+e_{3} v_{i, j+2}^{k-l}+e_{3} v_{i, j-2}^{k-l}+e_{3} v_{i+2, j}^{k-l} \\
& \left.+e_{3} v_{i-2, j}^{k-l}\right] \text {. }
\end{aligned}
$$

The last equation shows the HOC scheme for 2-D time fractional diffusion equation, where $a_{i^{\prime} s}, b_{i^{\prime} s}, \quad c_{i^{\prime} s}, \quad d_{i^{\prime} s}$ and $e_{i^{\prime} s}$ for $i=0,1,2,3$ are the coefficient which are defined as follows,

$$
\begin{aligned}
& a_{0}=\frac{250+1200 \mu_{0}-15 \mu_{0} h^{2}}{30}, \\
& b_{0}=\frac{150-1440 \mu_{0}+27 \mu_{0} h^{2}}{180}, c_{0}=\frac{5-120 \mu_{0}+2 \mu_{0} h^{2}}{60}, d_{0} \\
& =\frac{-5 \mu_{0} h^{2}}{120} \text {, } \\
& e_{0}=\left(-\frac{\mu_{0} h^{2}}{240}\right), a_{1}=\frac{250-1200 \mu_{0} \lambda_{1}+15 \mu_{0} \lambda_{1} h^{2}}{30}, \\
& b_{1}=\frac{150+1440 \mu_{0} \lambda_{1}-27 \mu_{0} \lambda_{1} h^{2}}{180}, \\
& c_{1}=\frac{5+120 \mu_{0} \lambda_{1}-2 \mu_{0} \lambda_{1} h^{2}}{60}, d_{1}=\frac{5 \mu_{0} \lambda_{1} h^{2}}{120}, \\
& e_{1}=\left(\frac{\mu_{0} \lambda_{1} h^{2}}{240}\right), a_{2}=\frac{25}{3}, b_{2}=\frac{5}{6}, c_{2}=\frac{1}{12}, \\
& a_{3}=\frac{-400+5 h^{2}}{120}, b_{3}=\frac{960-17 h^{2}}{1440}, \\
& c_{3}=\frac{60-h^{2}}{360}, d_{3}=\frac{5 h^{2}}{1440}, e_{3}=\frac{h^{2}}{2880} \text {. }
\end{aligned}
$$

\subsection{Matrix form of the Model problem.}

Matrix form of the numerical scheme for the proposed model is given below,

$$
\begin{aligned}
& \dot{A} V^{1}=\dot{B}_{0} \quad V^{0}+F^{1} \text { for } \quad k=1 \\
& \dot{A} V^{k}=\sum_{l=0}^{k-1} \quad B_{l} \quad V^{l}+F^{k}, \text { for } k=2,3 \ldots N .
\end{aligned}
$$


Clearly if we take $k=1$ the summation term will does not exists in equation (11) and we will get equation (12), and if $k=2,3, \ldots N$ then we get equation (13), In above equation (12) and (13) $\dot{A}, \dot{B}_{0}$ and $B_{l}$ are block pentadiagonal matrices of order $N_{x}+1 \times N_{y}+1$ and each block has fivepoint stencil.

\subsection{Additive Operating Splitting Method.}

As in AOS scheme the operator are applied independently, that is the AOS methods divides the $n$-dimensional space operator into a sum of $n$ onedimensional space discretization's. therefore, we take the first of two onedimensional problems. Before averaging the two solutions first we solve the system of equations in the $\mathrm{x}, \mathrm{y}$ directions respectively.

\section{RESULTS AND DISCUSSIONS}

\section{$2.1 \quad$ Test Problem 1}

Let us take the following PDE with the source term as in [10]

$\frac{\partial^{\gamma} \mathrm{v}(\mathrm{t}, \mathrm{x}, \mathrm{y})}{\partial \mathrm{t}^{\gamma}}={ }_{0} D_{t}^{1-\gamma}\left[\frac{\partial^{2} \mathrm{v}}{\partial x^{2}}+\frac{\partial^{2} \mathrm{v}}{\partial y^{2}}\right]$

$+e^{x+y}\left((1+\gamma) t^{\gamma}-\frac{\Gamma(2+\gamma) t^{2 \gamma}}{\Gamma(1+2 \gamma)}\right) . \quad t \in(0,1], \quad(x, y) \in(0,1]$

$v\left(t, 0, y_{1}\right)=e^{y} t^{1+\gamma}, v(t, x, y)=,e^{1+y} t^{1+\gamma}$,

$v(t, x, 0)=e^{x} t^{1+\gamma}, v(t, x, 1)=e^{1+x} t^{1+\gamma}$,

$v(0, x, y)=0, \quad t \in[0,1]$

$$
(x, y) \in(0,1]
$$

$v(t, x, y)=e^{x+y} y^{1+\gamma}$ representsthe analytical solution of the given test problem 1 .

\subsection{Test Problem 2}

Let's imagine another PDE with a source term as in [26]

$\frac{\partial^{\gamma} \mathrm{v}(\mathrm{t}, \mathrm{x}, \mathrm{y})}{\partial \mathrm{t}^{\gamma}}={ }_{0} D_{t}^{1-\gamma}\left[\frac{\partial^{2} \mathrm{v}}{\partial x^{2}}+\frac{\partial^{2} \mathrm{v}}{\partial y^{2}}\right]$

$+e^{-t} \sin (4 \pi x) \sin (4 \pi y)\left(-1-\frac{32 \pi^{2} \mathrm{t}^{\gamma}+\gamma \mathrm{t}^{\gamma-1}}{\Gamma(\gamma+1)}\right)$.

$t \in(0,1], \quad(x, y) \in(0,1] v(t, 0, y)=0$,

$v(t, 1, y)=0, v(t, x, 0)=0$,

$v(t, x, 1)=0, v(0, x, y)=\sin (4 \pi x) \sin (4 \pi y)$,

$(x, y) \in[0,1], \quad t \in[0,1]$

$v(t, x, y)=e^{-t} \sin (4 \pi x) \sin (4 \pi y)$, is the analytical solution of the given test problem 2 .

\subsection{Test Problem 3}

Consider the following PDE with a source term as in [27]

$\frac{\partial^{\gamma} \mathrm{v}(\mathrm{t}, \mathrm{x}, \mathrm{y})}{\partial \mathrm{t}^{\gamma}}={ }_{0} D_{t}^{1-\gamma}\left[\frac{\partial^{2} \mathrm{v}}{\partial x^{2}}+\frac{\partial^{2} \mathrm{v}}{\partial y^{2}}\right]$

$+e^{x+y+2 t}\left(1+\frac{\mathrm{t}^{\gamma}+\gamma \mathrm{t}^{\gamma-1}}{\Gamma(\gamma+1)}\right) ., \quad t \in(0,1]$

$(x, y) \in(0,1]$

$v(t, 0, y)=e^{y+2 t}, \quad v(t, 1, y)=e^{1+y+2 t}$,

$v(t, x, 0)=,e^{x+2 t}, v(t, x, 1)=e^{1+x+2 t}$,

$v(0, x, y)=e^{x+y}, \quad t \in[0,1]$

$(x, y) \in[0,1]$

$v(t, x, y)=e^{x+y+2 t}$, is the analytical solution.
Now using Riemann-Liouville in sense of fractional integral we have,

$$
{ }_{0} D_{t}^{-\gamma} t^{v}=\frac{\Gamma(1+v)}{\Gamma(v+\alpha+1)} t^{\alpha+v} \text { for } \alpha>0
$$

Using the equation (15) we can solve the problem.

The error norm $\left(l^{2}\right.$-norm) of our proposed HOC method is first order accurate in time and six order space Lets imagine that the error of the planned scheme satisfies,

$\left\|e^{k}\right\|_{l^{2}}=\left(\sum_{i=1}^{M-1}\left|e_{i, j}^{k}\right|^{2} h\right)=s_{1}(u) \tau+s_{2}(u) h^{6}$

Where $e_{i, j}^{k}=v_{i, j}^{k}-V_{i, j}^{k}$ and $s_{1}(u), s_{2}(u)$ depend on the analytical solution of $u$. The error decreases when we decrease the size $h$ and value of $\tau$. To evaluate the convergence order of our proposed method defined in [28], plugh $=\tau=\frac{1}{4}$ and halving the grid size. We see that the error is decreasing regularly, the results for $\left\|e^{k}\right\|_{l^{2}}$ and the convergence rate are given in Table 1, Table 2 and Table 3.

\section{CONCLUSIONS}

Higher order CFDM is applied to discretize the 2-D time fractional diffusion equation and its accuracy and stability is investigated. Higher order accuracy is the main advantage of CFD schemes. Here we have applied the AOS method to solve there sulting system efficiently. The resulting scheme consists of pentadiagonals that can be efficiently solved by AOS method and all the results are carried out completely. It is obvious that this algorithm has accuracy order one in time and sixth order with respect to space. Moreover our experimental results shows that the AOS scheme with HOC algorithm has the required accuracy. Figure 1, Figure 2 and Figure 3 shows the accuracy and convergence rate of our proposed scheme.

\section{REFERENCES}

[1] Metzler, R., Klafter, J. 2000. The random walk's guide to anomalous diffusion: a Fractional dynamics approach. Physics Reports, 339, 1-77.

[2] Lynch, V.E., Carreras, B.A., Castillo-Negrete, D., Ferreira-Mejias, K.M., Hicks, H.R. 2003. Numerical methods for the solution of partial differentia equations of fractional order. Journal of Computational Physics, 192, 406421.

[3] Meerschaert, M., Tadjeran, C. 2004. Finite difference approximations for fractional advection-dispersion flow equations. Journal of Computational and Applied Mathematics, 172, 65-77.

[4] Langlands, T.A.M., Henry, B.I. 2005. The accuracy and stability of an implicit solution method for the fractional diffusion equation. Journal of Computational Physics, 205 (3), 719-736.

[5] Yuste, S.B., Acedo, L. 2005. An explicit finite difference method and a new von Neumann-type stability analysis for fractional diffusion equations. SIAM Journal on Numerical Analysis, 42 (5), 1862-1874.

[6] Meerschaert, M.M., Scheffler, H.P., Tadjeran, C. 2006. Finite difference methods for two-dimensional fractional dispersion equation. Journal of Computational Physics, 211, 249-261.

[7] Tadjeran, C., Meerschaert, M.M. 2007. A second-order accurate numerical method for the two-dimensional fractional diffusion equation Journal of Computational Physics, 220, 813-823.

[8] Yuste, S.B. 2006. Weighted average finite difference methods for fractional diffusion equations. Journal of Computational Physics, 216, 264-274.

[9] Chen, C.M., Liu, F., Burrage, K. 2008. Finite difference methods and a fourier analysis for the fractional reaction-sub diffusion equation. Applied Mathematics and Computation, 198,754-769.

[10] Ghaffar, F., Islam, S., Badshah, N. Multigrid method with higher order compact finite difference method for fractional diffusion equation, Accepted.

[11] Ghaffar, F., Islam, S., Badshah, N. 2014. Multigrid method with higher order compact finite difference method for solution of 3D Helhmholtz equation. Abstract and Applied Analysis, Article ID 954658. 
[12] Ghaffar, F., Badshah, N., Khan, M.A., Islam, I. 2014. Multigrid method for 2D Helmholtz equation using higher order finite difference scheme accelerated by Krylove subspace. Journal of Applied Environmental and Biological Sciences, 4, 169-179.

[13] Ghaffar, F., Islam, I., Badshah, N. 2015. Multigrid method based on transformation free higher order scheme for solving 3D Helmholtz equation on nonuniform grids. Journal of Applied Environmental and Biological Sciences, 5,85-97.

[14] Ghaffar, F., Badshah, N., Islam, I., Khan, M. 2016. Multigrid method based on transformation free higher order scheme for solving 2D

Helmholtz equation on nonuniform grids. Journal of Dynamics and Differential Equations, 19. DOI 10.1186/s13662-016-0745-2.

[15] Du, R., Cao, W.R., Sun, Z.Z. 2010. A compact difference scheme for the fractional diffusion-wave equation. Applied Mathematical Modelling, 34, 2998-3007.

[16] Brunner, H., Ling, L., Yamamoto, M. 2010. Numerical simulations of 2D fractional sub diffusion problems. Journal of Computational Physics, $229,6613-6622$

[17] Hirsch, R.S. 1975. Higher order accurate difference solutions of fluid mechanics problems by a compact difference technique. Journal of Computational Physics, 24, 90-109.

[18] Lele, S.K. 1992. Compact finite difference schemes with spectral-like resolution. Journal of Computational Physics, 103, 16-42.

[19] Wang, H., Wang, K.X., Sircar, T. 2010. A direct O(Nlog2N) finite difference method for fractional diffusion equations. Journal of Computational Physics, 229, 8095-8104.

[20] Cui, M.R. 2009. Compact finite difference method for the fractional diffusion equation. Journal of Computational Physics, 228 (2), 7792-7804. [21] Sun, Z.Z., Li, X.L. 2005. A compact alternating direction implicit difference method for reaction diffusion equations. Math. Numer. Sinica, 27, 209-224. (inChinese).

[22] Cui, M.R. 2010. High order compact alternating direction implicit method for the generalized sine-Gordon equation. Journal of Computational and Applied Mathematics, 235, 837-849.

[23] Wang, H., Wang, K.X. 2011. An O(Nlog2N) alternating-direction finite difference method for two-dimensional fractional diffusion equations. Journal of Computational Physics, 230, 7830-7839.

[24] Gao, G.H.Z., Sun, Z.Z. 2011. A compact finite difference scheme for the fractional sub-diffusion equations. Journal of Computational Physics, $230,586-595$

[25] Cui, M. 2012. Compact alternating direction implicit method for twodimensional time fractional diffusion equation. Journal of Computational Physics, 231 (6), 2621-2633.

[26] Li, J., Tung Chen, Y.I. 2008. Computational Partial Differential Equation Using MATLAB. CRC Press New York.

[27] Siddiqui, M. 2010. Numerical Computation of Two-dimensional Diffusion Equation with Nonlocal Boundary Condition. International Journal of Applied Mathematics, 40,1, IJAM_40_1_04.

[28] Liao, W., Zhu, J., Khaliq, A.Q.M. 2002. An efficient high-order algorithm for solving systems of reaction-diffusion equations, Numer. Methods for Partial Differential Eq., 18, 340-354
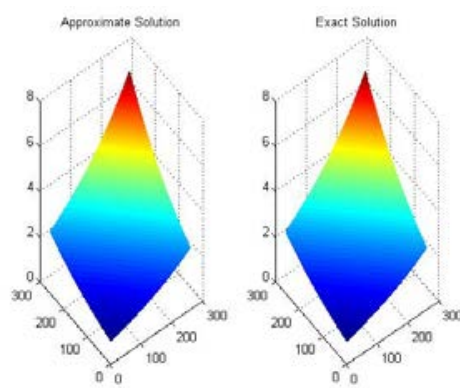

Figure 1: (Test Problem 1)

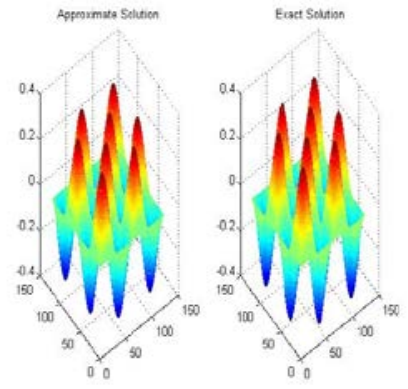

Figure 2: (Test Problem 2)

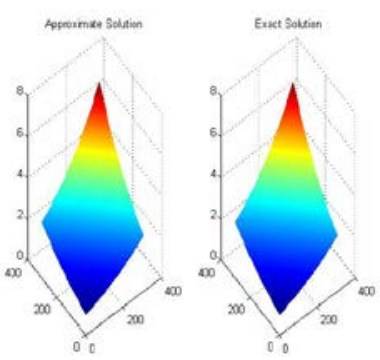

Figure 3: (Test Problem 3)

Table 1: (Test Problem 1)

Error norm, the accuracy order of the scheme for different value of $\gamma$.

\begin{tabular}{|c|c|c|c|}
\hline $\mathrm{h}$ & $\tau$ & $\|e\|_{l^{2}}$ & Order \\
\hline$\frac{1}{4}$ & $\frac{1}{4}$ & $4.3151 e^{-3}$ & ----- \\
\hline$\frac{1}{8}$ & $\frac{1}{8}$ & $2.0464 . e^{-4}$ & 0.0763 \\
\hline$\frac{1}{16}$ & $\frac{1}{16}$ & $4.0356 e^{-5}$ & 2.3422 \\
\hline$\frac{1}{32}$ & $\frac{1}{32}$ & $6.0877 e^{-6}$ & 2.7288 \\
\hline$\frac{1}{64}$ & $\frac{1}{64}$ & $7.6237 e^{-7}$ & 2.9973 \\
\hline$\frac{1}{128}$ & $\frac{1}{128}$ & $5.7646 e^{-8}$ & 3.7252 \\
\hline$\frac{1}{256}$ & $\frac{1}{256}$ & $1.4849 e^{-8}$ & 1.9569 \\
\hline
\end{tabular}

Table 2: (Test Problem 2)

Error norm, the accuracy order of the scheme for different value of $\gamma$.

\begin{tabular}{|c|c|c|c|}
\hline $\mathrm{h}$ & $\tau$ & $\|e\|_{l^{2}}$ & Order \\
\hline$\frac{1}{4}$ & $\frac{1}{4}$ & $6.800 e^{-3}$ & ---- \\
\hline$\frac{1}{8}$ & $\frac{1}{8}$ & $4.5763 . e^{-4}$ & 3.8933 \\
\hline$\frac{1}{16}$ & $\frac{1}{16}$ & $2.2629 e^{-5}$ & 4.3379 \\
\hline$\frac{1}{32}$ & $\frac{1}{32}$ & $3.2192 e^{-6}$ & 2.8134 \\
\hline$\frac{1}{64}$ & $\frac{1}{64}$ & $7.2640 e^{-7}$ & 2.1478 \\
\hline
\end{tabular}


Table 3: (Test Problem 3)

Error norm, the accuracy order of the scheme for different value of $\gamma$.

\begin{tabular}{|c|c|c|c|}
\hline $\mathrm{h}$ & $\tau$ & $\|e\|_{l^{2}}$ & Order \\
\hline$\frac{1}{4}$ & $\frac{1}{4}$ & $3.5100 e^{-2}$ & 0.9084 \\
\hline$\frac{1}{8}$ & $\frac{1}{8}$ & $1.8700 . e^{-2}$ & 1.7655 \\
\hline$\frac{1}{16}$ & $\frac{1}{16}$ & $5.500 e^{-3}$ & 4.5774 \\
\hline$\frac{1}{32}$ & $\frac{1}{32}$ & $2.3037 e^{-4}$ & 1.2911 \\
\hline$\frac{1}{150}$ & $\frac{1}{150}$ & $9.4135 e^{-5}$ & 3.5003 \\
\hline$\frac{1}{350}$ & $\frac{1}{350}$ & $8.3189 e^{-6}$ & 3.1202 \\
\hline$\frac{1}{1024}$ & $\frac{1}{1024}$ & $9.5671 e^{-7}$ & \\
\hline
\end{tabular}

